\title{
Distribution and linkage disequilibrium of the enhancer SNP rs5758550 among Latin American populations: influence of continental ancestry
}

\author{
Anna Beatriz Ribeiro Elias ${ }^{a}$, Gilderlanio Santana de Araújo ${ }^{b}$, \\ Sandro José de Souza ${ }^{c}$ and Guilherme Suarez-Kurtz ${ }^{\mathrm{a}, \mathrm{d}}$
}

\begin{abstract}
Objectives A single nucleotide polymorphism (SNP), rs5758550, in a critical enhancer region downstream of the CYP2D6 promoter was proposed to modulate CYP2D6 activity, depending on its linkage disequilibrium (LD) with the common CYP2D6 SNP, rs16947. We examined the influence of individual biogeographical ancestry on the frequency distribution of rs 5758550 and its LD with rs16947 in Latin American populations. We then inferred the impact of rs 5758550 on the predictive accuracy of
\end{abstract} CYP2D6 metabolizer status based on CYP2D6 haplotypes.

Methods The study cohorts consisted of the Admixed American (AMR) superpopulation of the 1000 Genomes Project $(n=347)$ plus an admixed Brazilian (BR) cohort $(\mathrm{N}=224)$. Individual proportions of Native, African and European ancestry estimated by ADMIXTURE analysis, were used to design four sub-cohorts, in which one of the three ancestral roots predominated largely ( $>6$ fold) over the other two: AMR-NAT and AMR-EUR, comprised 80 AMR individuals each, with $>70 \%$ Native or $>70 \%$ European ancestry, BR-EUR and BR-AFR comprised Brazilians with $>90 \%$ European $(n=80)$ or $>70 \%$ African ancestry $(n=64)$, respectively. CYP2D6 haplotypes were inferred based on 10 commonly reported CYPD6 variants with or without addition of the enhancer rs5758550 SNP, pairwise $L D$ was assessed by the $R^{2}$ parameter, and activity scores were used to infer CYP2D6 metabolizer status.

Results Minor allele frequency (MAF) of all CYP2D6 SNPs, except the rare $(<0.02)$ rs5030656 and rs35742688, differed significantly across sub-cohorts, whereas no difference was observed for rs 5758550 . The $R^{2}$ values for LD between rs 5758550 and $r 516947$ ranged from 0.15 (BR-AFR) to 0.85 (AMR-NAT), with intermediate values in the predominantly European sub-cohorts (0.34-0.67).

\section{Introduction}

Cytochrome P-450 2D6 (CYP2D6) is a major drug-metabolizing enzyme. The encoding gene, CYP2D6, presents high allele heterogeneity (https://www.pharmvar. org/gene/CYP2D6 [1]) that determines large inter-individual variation in enzyme activity, affecting the

Supplemental Digital Content is available for this article. Direct URL citations appear in the printed text and are provided in the HTML and PDF versions of this article on the journal's website, www.pharmacogeneticsandgenomics.com.
As a consequence, distribution of CYP2D6 haplotypes containing the rs16947 SNP plus rs5758550 wild-type (A) or variant (G) allele differed markedly across sub-cohorts. Comparison of the CYP2D6 activity scores assigned to the wild-type (CYP2D6*1) and the rs16947-containing haplotypes with or without inclusion of rs5758550, showed that knowledge of the rs 5758550 genotype has negligible impact on predicted CYP2D6 phenotypes in AMR-EUR and AMR-NAT, but affects prediction in 10.7 and $21.6 \%$ of BR-EUR and BR-AFR individuals, respectively.

Conclusion Collectively, the present results reveal potential pharmacogenomic (PGx) implications of the population diversity in Latin America, affecting a major drug-metabolizing pathway. Thus, the influence of enhancer rs 5758550 on assignment of CYP2D6 metabolic phenotypes varies markedly, according to the individual proportions of Native, European and African ancestry. This conclusion reinforces the notion that extrapolation of PGx data across the heterogeneous Latin American is risky, if not inappropriate. Pharmacogenetics and Genomics 30: 67-72 Copyright $\odot 2020$ Wolters Kluwer Health, Inc. All rights reserved.

Pharmacogenetics and Genomics 2020, 30:67-72

Keywords: biogeographical ancestry, CYP2D6, metabolic phenotypes, population diversity

${ }^{a}$ Coordenação de Pesquisa, Instituto Nacional do Câncer, Rio de Janeiro, 'Instituto de Ciências Biológicas, Universidade do Pará, Pará,

Instituto do Cérebro, UFRN, Rio Grande do Norte and dRede Nacional de Farmacogenética, Rio de Janeiro, Brazil

Correspondence to Guilherme Suarez-Kurtz, Coordenação de Pesquisa, Instituto Nacional de Câncer, Rua André Cavalcanti 37, Rio de Janeiro 20231-051, Brazil

Tel +55 213207 6552; e-mail: kurtz@inca.gov.br

Received 15 December 2019 Accepted 31 January 2020

pharmacokinetics and, indirectly, efficacy and toxicity of several commonly prescribed drugs. Pharmacogenetic (PGx) guidelines, such as those developed by the Clinical Pharmacogenetics Implementation Consortium (CPIC, https://cpicpgx.org/guidelines/) and the Dutch Pharmacogenetics Working Group (DPWG, https://www. pharmgkb.org/page/dpwg) base their recommendations for CYP2D6 substrates on genotype-inferred metabolic phenotypes. At present, commonly used genotyping panels comprise a limited number of known CYP2D6 variants 
(e.g. Affymetrix DMET Plus array) and supplemental copy number assessment. Metabolic activity scores [2] are assigned to each identified allele/haplotype, the activity score of the inferred diplotype is then calculated and used to infer the CYP2D6 metabolic phenotype. However, a single nucleotide polymorphism (SNP), rs5758550, in a critical enhancer region downstream of the CYP2D6 promoter was recently proposed to be a major determinant of CYP2D6 activity, depending on its linkage disequilibrium (LD) with the common CYP2D6 variant, rs16947 [3, 4]. The key SNP rs 16947 along with the rs 1135840 define the core haplotype CYP2D6*2, but this key SNP is also found with other variants on numerous other haplotypes (e.g. * $17, * 29, * 35$ and $* 41)$. The strength of LD between rs 16947 and rs5758550 was reported to vary considerably across populations of the 1000 Genomes Project, and thus impact to different degrees the accuracy of CYP2D6 genotyping panels to predict haplotype-phenotype associations globally [4]. We explored the impact of rs5758550 on the predicted CYP2D6 haplotype-phenotype association within the 1000 Genomes Admixed American (1KG AMR) superpopulation, comprised of individuals from the South American countries Colombia and Peru, from Puerto Rico as well as people of Mexican Ancestry living in Los Angeles, USA. Although Native American, European and African ancestry is shared among all four groups, the relative proportions of each ancestral root vary widely, reflecting the local dynamics and extent of admixture between the parental populations $[5,6]$. The resulting heterogeneity is a caveat against extrapolation of the $1 \mathrm{KG}$ AMR PGx data across Latin American peoples. Furthermore, Brazil, with the largest population in Latin America ( 210 million) is not represented in the $1 \mathrm{KG}$ AMR superpopulation. This led us to included data from an admixed Brazilian cohort and increase the scope of our analyses.

\section{Methods}

\section{CYP2D6 haplotype structure}

Genotype data for CYP2D6 and rs5758550 in the $1 \mathrm{KG}$ AMR superpopulation $(\mathrm{n}=347)$ were obtained from the $1 \mathrm{KG}$ Project phase 3 (https://grch37.ensembl.org/ info/index.html). CYP2D6 genotype data for Brazilians, self-identified as White $(n=80)$, Brown $(n=62)$ or Black $(\mathrm{n}=80)$ were derived from a previous study [7]. Allele discrimination at the rs5758550 locus in the Brazilian cohort was performed using a TaqMan assay. Individual diplotypes for the $1 \mathrm{KG}$ AMR and Brazilians were inferred from 10 commonly reported CYP2D6 variants (Supplementary Table 1, Supplemental digital content 1, http://links.lww.com/FPC/B365), with or without addition of the enhancer rs5758550 SNP, using the haplo-stats software, available at http://www.mayo.edu/research/ labs/statistical-genetics-genetic-epidemiology/software. This software attributes a posterior probability value for the diplotype configuration of each individual on the basis of estimated haplotype frequencies. The minimal posterior probability value for inclusion of an individual in our analyses was set at 0.8 . The SHEsis software platform (http://analysis.bio-x.cn/myAnalysis.php) was used for calculation of pairwise LD between loci. The extent of $L D$ is expressed by the $\mathrm{R}^{2}$ parameter.

\section{Assignment of activity scores to CYP2D6 haplotypes}

The activity scores assigned to the CYP2D6 star haplotypes by CPIC (https://www.pharmgkb.org/page/ cyp2d6RefMaterials) according to the standard CPICDPWG expert groups consensus method (Consensus AS; [8]) and the activity scores proposed by Ray et al. [4] comprising the rs 5758550 SNP were compared to assess the discordance in the predicted CYP2D6 metabolic phenotypes.

\section{Estimation of biogeographical ancestry}

The individual proportions of Native, European and African ancestry in the $1 \mathrm{KG}$ AMR superpopulation were estimated previously by unsupervised ADMIXTURE analysis at $K=3$ [9]. The choice of the three parental populations is based on the known demographic history of Latin America [5, 6]. The individual proportions of Native, European and African ancestry of Brazilians were available from our previous studies $[7,10]$.

\section{Statistics}

Allele and haplotype frequencies were derived by gene counting. The chi-square test was employed to assess deviations from Hardy-Weinberg equilibrium in each sub-cohort and for comparison of the frequency distribution of SNPs and haplotypes across sub-cohorts. Statistical significance was set at $P<0.05$.

\section{Results and discussion Diversity of biogeographical ancestry}

The Brazilian cohort and the 1KG AMR superpopulation had similar average proportions of European ancestry ( $56 \%$ vs. $50 \%$, respectively) but differed markedly ( $>5$-fold) with respect to African and Native ancestry, which were estimated to be, respectively, $36 \%$ and $7 \%$ in Brazilians vs. $8 \%$ and $42 \%$ in AMR (Table 1). These data support our strategy of combining Brazilian and $1 \mathrm{KG}$ AMR data to expand the scope of the present analyses of the impact of biogeographical ancestry on CYP2D6 haplotype structure. For this purpose, we devised sub-cohorts in which one of the three ancestral roots predominates largely ( $>6$-fold) over the other two. The $1 \mathrm{KG}$ AMR yielded two sub-cohorts, designated AMR-NAT and AMR-EUR, comprising 80 individuals each, with the highest proportions of either Native or European ancestry, respectively (Table 1 and Supplementary Figure 1, Supplemental digital content 2, http://links.lww.com/FPCl B366). The AMR-NAT sub-cohort includes 67 Peruvians (PEL), 12 individuals with Mexican Ancestry (MXL) and one Colombian (CLM), all of which had $>70 \%$ Native ancestry. The AMR-EUR sub-cohort was formed by 


\begin{tabular}{|c|c|c|c|c|c|}
\hline \multirow[b]{2}{*}{ Population } & \multirow[b]{2}{*}{ Cohort } & \multirow[b]{2}{*}{$\mathrm{N}$} & \multicolumn{3}{|c|}{ Biogeographical ancestry, mean (95\% Cl) } \\
\hline & & & European & Native & African \\
\hline \multirow{3}{*}{$1 \mathrm{KG}$ AMR } & Overall & 347 & $0.503(0.475-0.530)$ & $0.418(0.387-0.449)$ & $0.079(0.070-0.089)$ \\
\hline & AMR-NAT & 80 & $0.117(0.095-0.139)$ & $0.872(0.849-0.895)$ & $0.011(0.005-0.017)$ \\
\hline & AMR-EUR & 80 & $0.791(0.778-0.804)$ & $0.141(0.130-0.152)$ & $0.068(0.060-0.076)$ \\
\hline \multirow[t]{3}{*}{ Brazilians } & Overall & 224 & $0.563(0.521-0.604)$ & $0.074(0.063-0.084)$ & $0.363(0.325-0.402)$ \\
\hline & BR-EUR & 82 & $0.943(0.938-0.948)$ & $0.027(0.023-0.031)$ & $0.030(0.026-0.035)$ \\
\hline & BR-AFR & 62 & $0.135(0.116-0.154)$ & $0.068(0.055-0.081)$ & $0.797(0.777-0.816)$ \\
\hline
\end{tabular}

Fig. 1

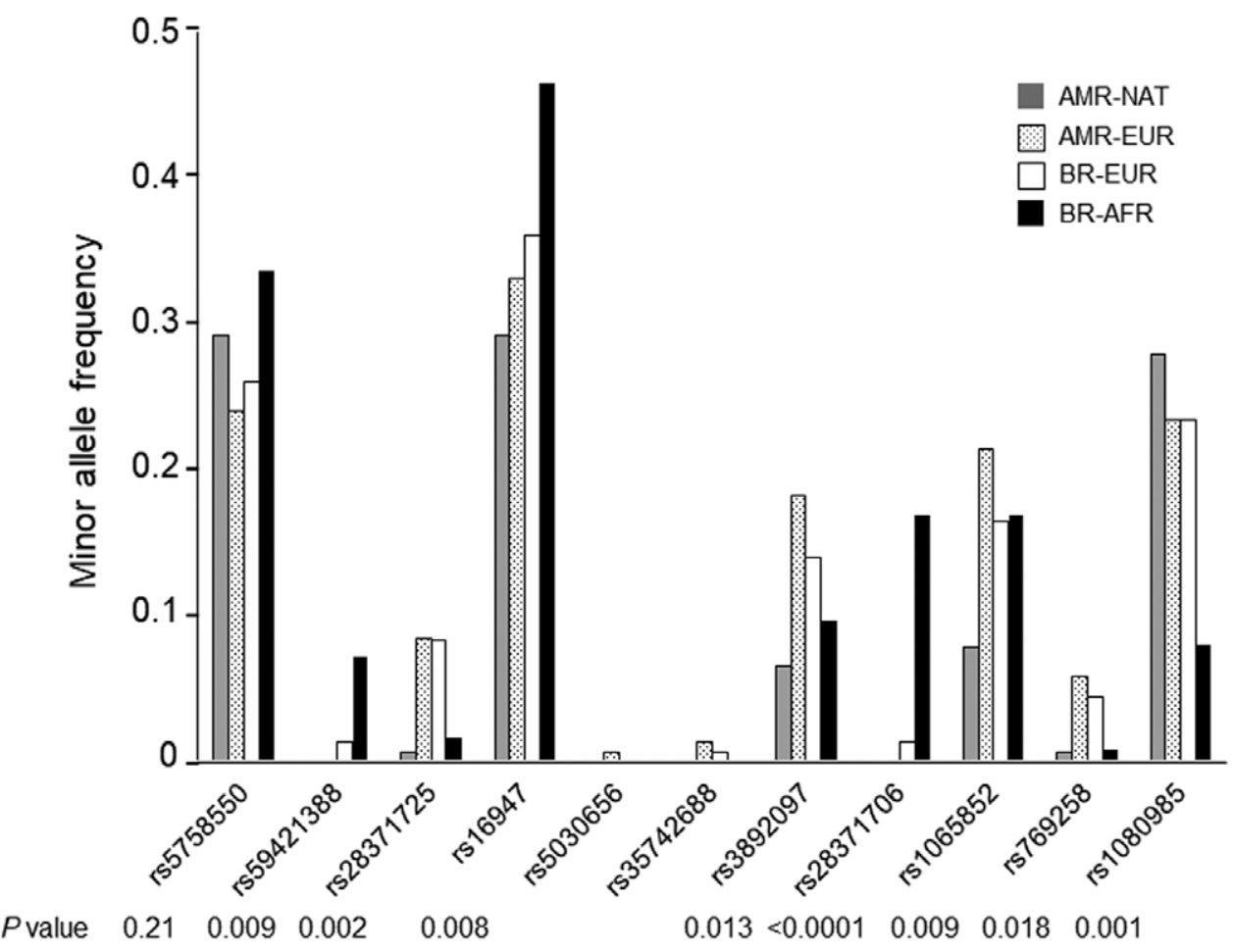

Minor allele frequency (MAF) of the interrogated CYP2C6 and the CYP2D6-enhancer rs5758550 single-nucleotide polymorphisms in the study sub-cohorts. The $P$-values are for chi-square tests for differences in allele frequency of each SNP among the sub-cohorts. SNP, single nucleotide polymorphism.

52 Puerto Ricans (PUR), 25 CLM and three MXL, all with $>70 \%$ European ancestry. The Brazilian sample also yielded two sub-cohorts, designated BR-AFR (62 self-reported Brown or Black individuals with $>70 \%$ African ancestry) and BR-EUR (80 self-reported White or Brown individuals with $>90 \%$ European ancestry). Because of the comparatively small contribution of African ancestry in the $1 \mathrm{KG}$ AMR and of Native ancestry in the Brazilian sample, AMR-AFR and BR-NAT sub-cohorts could not be formed.

\section{Distribution of rs 5758550 and $C Y P 2 D 6$ single nucleotide polymorphisms in the study sub-cohorts}

Figure 1 shows the minor allele frequency (MAF) of rs5758550 and CYP2D6 SNPs in the four study sub-cohorts. Distribution of rs5758550 genotypes complied with Hardy-Weinberg equilibrium in all sub-cohorts, MAF's ranged from 0.25 (AMR-EUR) to 0.33 (BR-AFR), but the difference across sub-cohorts did not reach statistical significance $(P=0.21)$. Significant differences in MAF, however, were observed for all CYP2D6 SNPs, except rs5030656 and rs35742688, both of which were absent or uncommon $(<2 \%)$ in the study sub-cohorts. The rs 16947 was the most common CYP2D6 SNP in all sub-cohorts, with MAF values ranging from 0.29 (AMRNAT) to 0.45 (BR-AFR). The lowest frequency in AMRNAT may be explained by the likely Asian origin of the first migrants into the American continent [5], since East Asians have the lowest MAF of rs 16947 among the $1 \mathrm{KG}$ superpopulations. The highest MAF in BR-AFR is consistent with their predominant African ancestry; accordingly, the highest MAF for the rs16947 among the $1 \mathrm{KG}$ 
superpopulations was observed in Africans (0.55). African ancestry may also explain the distinct distribution of the rs59421388, rs28371706 and rs1080985 SNPs in BR-AFR: the first two SNPs were detected in 7 and $16 \%$ of the BR-AFR individuals, respectively, but were absent in the $1 \mathrm{KG}$ AMR sub-cohorts and rare in BR-EUR (Fig. 1). By contrast, the minor rs 1080985 allele was $4-5$ times less common in BR-AFR than in the cohorts of predominant European of Native ancestry (Fig. 1).

For each studied sub-cohort, haplotype diagrams comprising rs 5758550 and $C Y P 2 D 6$ SNPs with MAF $>0.03$ are shown in Supplementary Figure 2 (Supplemental digital content 3, http://links.lww.com/FPC/B367). Of particular interest to the present study is the wide range of $R^{2}$ values (0.15-0.85) for LD between rs5758550 and rs 16947 , which most likely results from the predominance of a distinct ancestral root in each sub-cohort. Accordingly, comparison of the $\mathrm{R}^{2}$ values in the study sub-cohorts and the $1 \mathrm{KG}$ superpopulations reveals values of 0.15 and 0.11 for BR-AFR and $1 \mathrm{KG} A F R$, respectively, whereas, in the predominantly European sub-cohorts, $\mathrm{R}^{2}$ ranged between 0.41 (BR-EUR) and 0.67 (AMR-EUR), compared to 0.56 for $1 \mathrm{KG}$-EUR. The relatively lower $\mathrm{R}^{2}$ in BR-EUR may be tentatively ascribed to differences in local ancestry, that is, ancestry at specific loci vs. global ancestry, a recognized caveat in admixed populations [11].

\section{Impact of rs5758550 on the assignment of CYP2D6 metabolic phenotypes}

The variable extent of LD between rs16947 and rs5758550 among the study sub-cohorts implies that knowledge of rs5758550 genotype might affect to different degrees the predictive accuracy of translating CYP2D6 haplotypes into metabolic phenotypes among Latin Americans, according to the individual ancestry proportions. To explore this issue, we initially verified the distribution of CYP2D6 haplotypes containing the rs16947 SNP, namely *2, *17, *29, *35 and *41, plus rs5758550 wild-type (A) or variant (G) allele (Fig. 2). The proportion of CYP2D6*2 haplotypes comprising also the variant rs5758550 allele decreased from $~ 99 \%$ in AMRNAT and AMR-EUR to $80 \%$ in BR-EUR and to $42 \%$ in BR-AFR. The CYP2D6 *17 and CYP2D6*29 haplotypes were absent or rare $(1.2 \%)$ in the Native and European sub-cohorts, but present in $16 \%$ and $7.2 \%$ of BR-AFR, respectively. The $* 17$ haplotype associated mainly $(90 \%$ of cases) with the rs $5758550 \mathrm{G}$ allele, whereas rs $5758550 \mathrm{~A}$ was the predominant allele associated with the $* 29$ haplotype. The CYP2D6 *35 and CYP2D6*41 haplotypes were rare $(<1 \%)$ in Native and African sub-cohorts, but detected, respectively, at 4-6\% and $8 \%$ in the two EUR sub-cohorts. In AMR-EUR these haplotypes associated exclusively with a distinct rs5758550 allele: CYP2D6*35 with the $\mathrm{G}$ allele and CYP2D6*41 with the A allele. In BR-EUR, both rs5758550 alleles were found in association with CYP2D6*35 and CYP2D $6 * 41$.
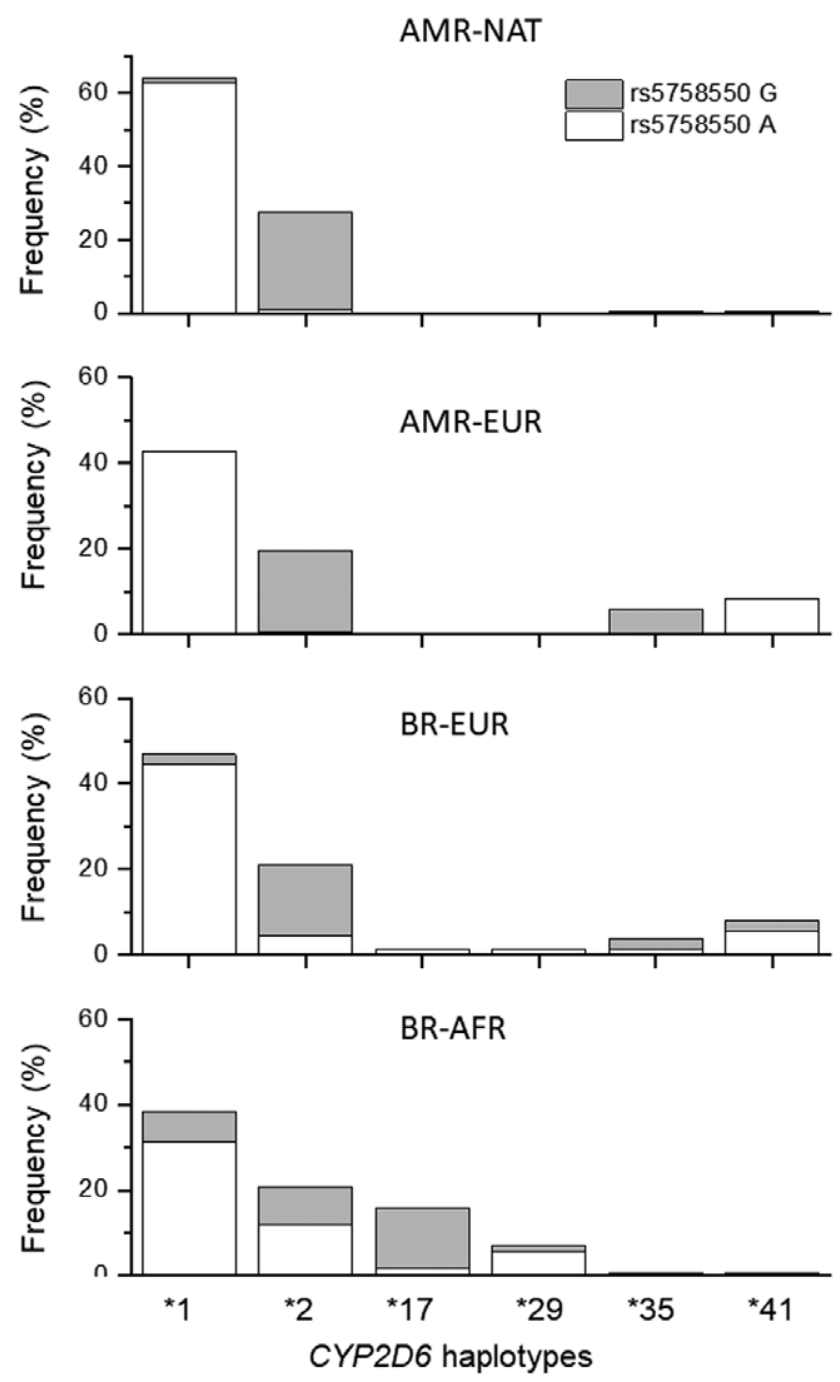

Frequency distribution of CYP2D6 haplotypes (star alleles) among the study sub-cohorts. Shown are the wild-type (default) CYP2D6*1 and haplotypes ( ${ }^{*} 2,{ }^{*} 17,{ }^{*} 29,{ }^{*} 35$ and ${ }^{*} 41$ ) containing the rs 16947 single-nucleotide polymorphism. Each column is partitioned in two segments corresponding to the haplotypes containing either the rs5758550 A (white) or G (grey) allele.

In an attempt to estimate the impact of rs5758550 on the predictive accuracy of translating CYP2D6 haplotypes into metabolic phenotypes in the different sub-cohorts, we compared the Consensus AS assigned to the star haplotypes (without rs5758550) to the activity scores proposed by Ray et al. [4] for combinations of rs5758550 and rs 16947 (Table 2). Discordant activity scores applied to CYP2D6*2 and CYP2D6*17 when associated with wildtype rs5758550A allele (lower metabolic activity compared to Consensus AS for *2 and *17, respectively), and possibly to CYP2D6*41 when associated with the variant rs $5758550 \mathrm{G}$ allele (higher metabolic activity compared to Consensus AS for *41). As shown in Fig. 3, the summed 
Table 2 Assignment of activity scores to CYP2D6 haplotypes

\begin{tabular}{|c|c|c|c|c|c|}
\hline \multicolumn{2}{|c|}{ CPIC-DPWG consensus [8] } & \multicolumn{4}{|c|}{ Ray et al. [4] } \\
\hline & Activity & Haplotype & Activity & Haplotype & Activity \\
\hline Haplotype & Score & rs5758550 A & Score & rs5758550 G & Score \\
\hline *1 & 1 & $\mathrm{n} / \mathrm{n}$ & 1 & $\mathrm{H} 1 \mathrm{a}$ & 2 \\
\hline *2 & 1 & НЗа & 0.5 & $\mathrm{H} 2 \mathrm{a}$ & $1-1.5$ \\
\hline *17 & 0.5 & $\mathrm{H} 3 \mathrm{c}$ & $<0.5$ & $\mathrm{H} 2 \mathrm{~b}$ & 0.5 \\
\hline *41 & 0.5 & $\mathrm{n} / \mathrm{n}$ & 0.5 & $\mathrm{n} / \mathrm{n}$ & $>0.5$ \\
\hline
\end{tabular}

Discordant activity scores between CPIC-DPWG Consensus [8] and Ray et al. [4] are highlighted in bold.

$\mathrm{n} / \mathrm{n}$, no name in Table 2 of Ray et al. [4]

Fig. 3

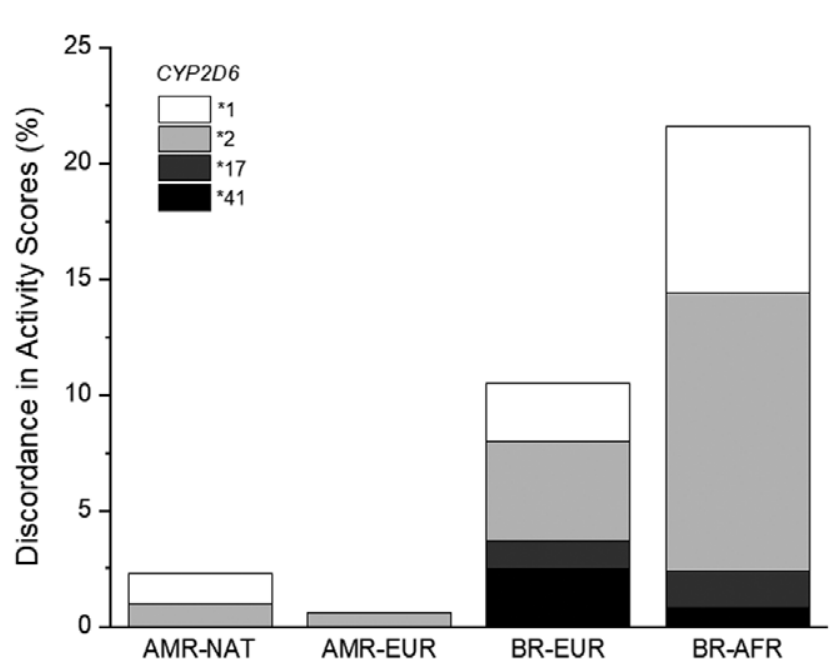

Discordance in activity scores assigned to CYP2D $6^{*}$ haplotypes by Ray et al. [4], which include the rs5758550 SNP, versus the Consensus AS proposed by CPIC-DPWG [8], which do not include rs5758550. The columns show the percentage of discordance in each sub-cohort for the CYP2D6*1, ${ }^{*} 2,{ }^{*} 17$ and ${ }^{*} 41$ haplotypes. SNP, single nucleotide polymorphism.

discordance in CYP2D6*2, *17 and *41 ranged from $<1 \%$ in AMR-NAT and AMR-EUR, to $8.2 \%$ in BR-EUR, and to $14.4 \%$ in BR-AFR. An additional source of discordance between the two activity scores systems is the default wild-type haplotype CYP2D $6 *$, which was assigned an activity score $>1$ when associated with rs $5758550 \mathrm{G}$ allele [4]. Inclusion of CYP2D6*1 has little $(<1 \%)$ impact on activity score discordance in AMR-EUR and AMR-NAT, but adds another 2.5 and $7.2 \%$ to the extent of discordance in BR-EUR and BR-AFR, thus affecting 10.7 and $21.6 \%$ of inferred phenotypes in these Brazilian sub-cohorts, respectively.

Collectively, the present results reveal novel PGx implications of the population diversity in Latin America, affecting a major drug-metabolizing pathway. These results reinforce the notion that extrapolation of $\mathrm{PGx}$ data across the heterogeneous Latin American is risky, if not inappropriate [12], and suggest that adequate inference of CYP2D6 metabolizer phenotype in Latin Americans, especially those with predominant African ancestry, may benefit from knowledge of the rs 5758550 genotype. Nevertheless, we acknowledge that the clinical significance of the enhancer SNP has not been established. Indeed, correction for the presence or absence of the enhancer SNP did not lead to improved prediction of endoxifen levels in breast cancer patients [13], while genotyping for the enhancer SNP did not account for the wide variability in systemic exposure to atomoxetine, a CYP2D6 substrate, in a relatively small cohort of children with attention-deficit/hyperactivity disorder [14]. We also acknowledge as a limitation of this study that CYP2D6 haplotypes were inferred statistically, based on $10 \mathrm{com}$ mon SNPs, and at least one key variant, namely rs 1135840 (NG_008376.3:g.4181G>C) was not interrogated. Other potentially functional variants as well as copy number variation, not considered in our analyses, may affect the activity score assignment and thus the estimated impact of rs5758550 on predicted CYP2D6 metabolic activity.

In conclusion, we showed that addition of the enhancer rs5758550 to CYP2D6 genotyping panels has a potential effect on the prediction of CYP2D6 metabolic phenotypes in Latin American peoples. The extent of this effect depends on the individual proportions of Native, European and African ancestry: it is negligible in persons of predominant Native ancestry, affects 1\% (AMREUR) and 11\% (BR-EUR) of persons of predominant European ancestry, and $>20 \%$ of Brazilians with predominant African ancestry. The considerable larger effect in BR-EUR than in AMR-EUR may be ascribed to differences in local ancestry, that is, ancestry at specific loci vs. global ancestry, especially African ancestry, which is, on average, five-fold higher in BR-EUR than in AMR-EUR.

\section{Acknowledgements}

Research in the authors' labs is supported by Brazilian agencies Conselho Nacional de Desenvolvimento Científico e Tecnológico (CNPq), Fundação de Amparo à Pesquisa do Estado do Rio de Janeiro (Faperj) and Departamento de Ciência e Tecnologia (Decit), Ministry of Health. 


\section{Conflicts of interest}

There are no conflicts of interest.

\section{References}

1 Gaedigk A, Ingelman-Sundberg M, Miller NA, Leeder JS, Whirl-Carrillo $\mathrm{M}$, Klein TE; PharmVar Steering Committee. The pharmacogene variation (pharmvar) consortium: incorporation of the human cytochrome P450 (CYP) allele nomenclature database. Clin Pharmacol Ther 2018; 103:399-401.

2 Gaedigk A, Simon SD, Pearce RE, Bradford LD, Kennedy MJ, Leeder JS. The CYP2D6 activity score: translating genotype information into a qualitative measure of phenotype. Clin Pharmacol Ther 2008; 83:234-242.

3 Wang D, Poi MJ, Sun X, Gaedigk A, Leeder JS, Sadee W. Common CYP2D6 polymorphisms affecting alternative splicing and transcription: long-range haplotypes with two regulatory variants modulate CYP2D6 activity. Hum Mol Genet 2014; 23:268-278.

4 Ray B, Ozcagli E, Sadee W, Wang D. CYP2D6 haplotypes with enhancer single-nucleotide polymorphism rs5758550 and rs16947 ( ${ }^{\star} 2$ allele): implications for CYP2D6 genotyping panels. Pharmacogenet Genomics 2019; 29:39-47.

5 Salzano FM. The evolution of science in a Latin-American country: genetics and genomics in brazil. Genetics 2018; 208:823-832.

6 Ruiz-Linares A, Adhikari K, Acuña-Alonzo V, Quinto-Sanchez M, Jaramillo C, Arias $\mathrm{W}$, et al. Admixture in Latin America: geographic structure, phenotypic diversity and self-perception of ancestry based on 7,342 individuals. Plos Genet 2014; 10:e1004572.
7 Friedrich DC, Genro JP, Sortica VA, Suarez-Kurtz G, de Moraes ME, Pena $\mathrm{SD}$, et al. Distribution of CYP2D6 alleles and phenotypes in the Brazilian population. PLoS One 2014; 9:e110691.

8 Caudle KE, Sangkuhl K, Whirl-Carrillo M, Swen JJ, Haidar CE, Klein TE, et al. Standardizing CYP2D6 genotype to phenotype translation: consensus recommendations from the Clinical Pharmacogenetics Implementation Consortium and Dutch Pharmacogenetics Working Group. Clin Trans/ Sci 2020; 13:116-124.

9 Suarez-Kurtz G, Araújo GS, de Sousa SJ. Pharmacogeomic implications of population diversity in Latin America: TPMT and NUDT15 polymorphisms and thiopurine dosing. Pharmacogenet Genomics 2020; 30:1-4.

10 Pena SD, Di Pietro G, Fuchshuber-Moraes M, Genro JP, Hutz MH, Kehdy FdeS, et al. The genomic ancestry of individuals from different geographical regions of Brazil is more uniform than expected. PLoS One 2011; 6:e17063.

11 Price AL, Tandon A, Patterson N, Barnes KC, Rafaels N, Ruczinski I, et al. Sensitive detection of chromosomal segments of distinct ancestry in admixed populations. Plos Genet 2009; 5:e1000519.

12 Suarez-Kurtz G, Parra EJ. Population diversity in pharmacogenetics: a Latin American perspective. Adv Pharmacol 2018; 83:133-154.

13 Sanchez-Spitman AB, Moes DA, Gelderblom H, Dezentjé VO, Swen J J, Guchelaar HJ. The effect of rs5758550 on CYP2D6*2 phenotype and formation of endoxifen in breast cancer patients using tamoxifen. Pharmacogenomics 2017; 18:1125-1132.

14 Brown JT, Abdel-Rahman SM, van Haandel L, Gaedigk A, Lin YS, Leeder JS. Single dose, CYP2D6 genotype-stratified pharmacokinetic study of atomoxetine in children with ADHD. Clin Pharmacol Ther 2016; 99:642-650. 